\title{
The Family Doctor and the Family Nurse
}

\author{
G. SWIFT,* M.A., B.M., B.CH. ; I. A. MACDOUGALL,† M.B.E., M.R.C.S., L.R.C.P., D.P.H.
}

Brit. med. F., 1964, 1, 1697-1699

The following is an account of the attachment of nurses, midwives, and health visitors by a local health authority to work exclusively with family doctors. It is set out in two partsthe first, written by a family doctor, describes the method of working and its advantages over what we in Hampshire have now come to regard as the old method whereby nurses, midwives, and health visitors work " on the district." The second part is designed briefly to indicate the administrative procedures for making available a nurse-attachment scheme to family doctors.

\section{Part I. Practice}

This is a partnership of six doctors working largely in the National Health Service, with a list of about 17,000 and some private work. All the work is done from the same premises; there are six consulting-rooms, two waiting-rooms, office accommodation, and a flat with two resident receptionists, which enables the partnership to run a 24-hour switchboard service. National Health patients live up to 7 miles $(11 \mathrm{~km}$.) from the surgery.

From 1958 to 1961 we had one health visitor, and since 1961 we have had two health visitors; in 1961 two district nurses and two midwives were also attached to the practice. This later attachment was in fact no new experience to us, as up to 1948 we had ourselves employed two nurse/midwives to work with our private patients.

The principles upon which we work are as follows:

(1) The attached staff are accepted as full colleagues-that is to say, they are expected to carry out their own proper duties in their own field of employment as they would if employed in the traditional way by a local health authority. They are not in any way directed in what they do, but rather their help is enlisted when required. The relationship might in some ways be compared to that of a consultant to his ward sister.

(2) They have full use of our premises and have facilities for compiling and keeping their records on surgery premises. Their messages, whether written or by telephone, are recorded for them by the receptionists in the same way as for the partners, and they use the consulting-rooms and waiting-rooms as required.

(3) They work with, and only with, the patients in the practice list, except for the occasional relief work and a few school clinics when necessary.

(4) Because of the scattered nature of the practice it is essential that all the attached staff have their own cars; these are provided by the local health authority.

In general practice, except in small enclosed rural communities, the health visitor, district nurse, and midwife tend to be rather impersonal entities. They are infrequently seen by the general practitioner, and communication tends to be by telephone, or by written or even verbal messages, which may lead to misunderstanding. Often in large towns, for instance, one general practitioner is associated with as many as 20 mem-

* General Practitioner, Winchester.

† County Medical Officer, Hampshire. bers of the nursing staff of the local health authority, and the converse holds as well. We have found that under our system the opportunity to work closely and by personal contact with a few attached nursing staff has been of enormous benefit. The habit of meeting regularly leads to easy exchange of information, which as mutual confidence increases can, if necessary, be critical. Small items of information about the family background or rather intangible clinical impressions on the part of the nurse can be reported to the doctors without fear of ridicule. Thus 10 o'clock in the morning, when all the partners and all the attached staff are present in the surgery, is a most important time when information and requests for help can be informally passed from one to the other. It is fair to say that the doctors, health visitors, district nurses, and midwives all feel that by this continued meeting and co-operation they are able to give patients a better service.

From the practice administration point of view it is inevitable that the overheads should rise somewhat owing to the expense of the telephones, extra work for the receptionists, and general wear and tear on the premises.

\section{Health Visitors}

Before our first health visitors were attached in 1958 we were somewhat mistrustful of their profession, but we have now achieved extremely good co-operation because we work with them and understand fully the nature and requirements of their work. We know what can and should be done by a health visitor and that her contribution to general medical care is complementary to that of a general practitioner.

Infant Welfare.-The hospitals, when reporting recent confinements to the local health authority, also send the name of the patient's general practitioner. In this way it is simple for all cases that are the responsibility of the practice to be notified to the practice health visitors. On Monday afternoons the health visitors have the use of a large waiting-room, where they carry out an infant-welfare clinic largely confined to routines of weighing, queries on feeding, and so on. On Wednesday they hold a further clinic when one of the partners is present in his consulting-room. At this clinic most of the routine immunizations are carried out and children are seen with minor ailments by the general practitioner, and joint consultations take place between the health visitor and the general practitioner in selected cases. In this way a joint plan of action is decided upon, the mother gets unified advice on the treatment of her child at one visit and there is little or no danger of her being confused by conflicting advice. We believe that by this method we are able to pick out the borderline cases which require referring to the paediatrician, and, what is more important, to avoid unnecessary referral to the hospital. It is easy to arrange for follow-up of children who have been seriously ill or psychologically maladjusted. The health visitors find benefit from having access at all times to the general practitioner's personal records and hospital letters, 
and the general practitioners at ordinary surgery find it valuable to have a health visitor's progress report available when required.

Young People.-The health visitors' work is by no means confined to child welfare. They support us in the help of young persons, such as those who are maladjusted at school or at home. They assist with the delinquent or, more important, in the prevention of delinquency and in the problems of illegitimacy.

Elderly.-The health visitors are doing an increasing amount of work with our geriatric cases in helping with the problems of the elderly and making detailed arrangements for home help, chiropody, meals on wheels, etc.

In all age-groups they assist the partners and save them a great deal of time by acting as liaison officers with all the various departments of the local authority and the local branches of the central government departments.

\section{Midwives}

When the midwives were originally attached to us their great fear was that they would be down-graded to maternity nurses and lose their professional standing. We are able to report that this has in no way happened, and they feel that by attachment to a small group of doctors they can do more satisfying work than before.

The midwives attached to the practice deal with all cases for domiciliary confinement within the practice and with no other cases except in emergencies.

On Tuesday afternoons they hold their own antenatal clinic in the surgery, and another is held, sometimes on a Friday, if the numbers are sufficient. Originally we thought that it might be of benefit to hold one of these as a joint clinic with midwives and doctors both present, but after a short experience we decided against this. We think that the expectant mother wishes to see the family doctor and midwife for slightly different reasons and is likely to pose different problems to both of them. She will do this better if she sees them separately; if she sees them both together her problems are likely to be unsolved. Instead we are sure that the best means of cooperation between midwives and doctors is, firstly, by personal daily contact, when any question can be raised, and, secondly, by the use of a maternity co-operation card on which both make notes when the patient is seen; this card is carried by the mother to all consultations, whether with the midwife, the doctor, or at the hospital. The appointments are made by the receptionists, who arrange for visits to midwife and family doctor alternately.

\section{District Nurses}

The district nurses do exactly the same work as they would if not attached to the practice, but we have found that there is a real advantage in frequent meetings between doctors and nurses and the exchange of information. From the doctors' point of view this sometimes saves an unnecessary visit to the patient, as the nurse can take messages and report on the patient's condition. It is also easy to arrange to meet at an agreed time at a patient's house, when the dressings can be taken down and the wound examined; some cases are dressed at the surgery by the nurses and seen together with the doctor.

The treatment of cases by the nurses at the surgery should be developed in the future; there are a considerable number of patients who could attend for dressings, by appointment, thus saving travelling by the nurses. Unfortunately, owing to lack of accommodation, we have not been able to develop this as much as we should like, but if we had a treatment-room that the nurses could use it would be of great benefit to patients, nurses, and doctors alike. With such a treatment-room it is possible to envisage that we should be able to do more in the way of minor operations, because the nurses would be there to prepare the room, instruments, dressings, and so on.

\section{Part II. Administrative}

It is now several years since the practice was adopted in this county of attaching nurses, midwives, and health visitors to work exclusively with family doctors, either in single-handed practice or in partnership. So successful has this method proved that it is felt that a brief account written from the administrator's angle might be of interest in other areas contemplating the introduction of such an arrangement.

The enormous advantages resulting from the attaching of local health authority nursing staff to work with family doctors is clearly indicated in the preceding account, and indeed much has already been written on the subject by other authors. But it is here in Hampshire that this method of working is most extensively used, and naturally the experience gained is very considerable. The subcommittee of the Standing Medical Advisory Committee of the Central Health Services Council in preparing its report took evidence from this county, among others, and it is most gratifying to note that this method of attaching nursing staff to work with general practitioners is clearly advocated (Gillie Report, paras 60 and 138).

There are now about 150 family doctors in this county who have attached nursing staff working exclusively with them in their practices, and so far I have yet to meet one of these doctors, or indeed one of the nurses, midwives, or health visitors, who would wish to put the clock back and return to the old traditional methods. The doctors for their part find that they can now give full and comprehensive care to their patients in a way never possible before ; and as for the nurses, they infinitely prefer to work under the clinical direction of the family doctor and as a member of a team rather than the isolation of working on the district. Not surprisingly the patients themselves become rapidly aware of the distinct benefit in the medical service resulting from their family doctor having his own team of nurses.

When the scheme was first started in Hampshire it was thought from the administrative angle that once it got well under way there might be a need considerably to increase the establishment of county nursing staff. This has certainly not proved the case, and with now over 100 nurses, midwives, and health visitors attached to family doctors it can still be said that no increase in establishment has had to be asked for solely on account of operating an attachment scheme. Naturally one finds that with certain practices an attached nurse may spend more time travelling than when, under the old arrangement, she had a tidy circumscribed district to work, and, although this may mean that her mileage claim submitted may be higher, her actual working-time throughout the day is more economically spent as a result of planning her work with her doctors. All nurses, midwives, and health visitors have, of course, motor-cars, either their own or provided by the county, and any increase in their mileage claims as a result of working with family doctors is unquestionably accepted by the county as being a small price to pay for a much improved medical service.

\section{County Nursing Staff}

The county nursing staff is made up of nurses who practise as district nurses, as midwives, or as health visitors, or do a combination of these duties-for example, nursing and midwifery, or nursing, midwifery, and health visiting; the latter we refer to as a triple-purpose nurse. We find that the triplepurpose nurse is the ideal ally to attach to the single-handed 
rural practitioner, for she can well handle the average case-load of such a practice so far as district nursing, midwifery, and health visiting are concerned. In urban areas we favour the use of the nurse/midwife and prefer that the health visiting is done by a nurse practising solely as such. In assessing the nursing needs of a practice the following rule of thumb is now followed, and is found to work well: (a) one nurse practising as a district-nurse/midwife can serve a population of $3,500-$ 4,000 patients ; (b) one nurse practising solely midwifery can attend 60-70 domiciliary confinements in a year; and $(c)$ one nurse practising solely as a health visitor can serve a population of $5,000-6,000$, which on average contains 500 children in the $0-5$ age-group.

The question of whether a family doctor or group of doctors should have attached staff is naturally left to them to decide, and if the practice expresses an interest in having this type of assistance the county medical officer and the county nursing officer would meet the doctors concerned and fully discuss the proposal with them, explaining certain fundamental factors which as a result of experience have been found essential for an attachment scheme to prosper:' for example, it is essential for the nurses to be accepted by the doctors as full members of the practice team. There must be some fixed time of the day when the nurses can meet the doctors and exchange information regarding cases or visits required. The mechanism by which the nurses will make contact with the doctors and with midwives and health visitors is a vital factor. Almost every surgery varies in this respect according to the pattern on which the surgeries are held. In some cases all the nurses meet the general practitioners before they start out on their rounds, leaving a list of addresses at which they are to be found during the morning, and then all meet again in the afternoon. In other cases the nurses go straight out and then return midmorning to see the general practitioners when the surgery is finished. Speaking from experience, I would say that it does not matter at all how this is done so long as a definite pattern of contact is established. There must be free access of nurses to doctor's records, as is the case between the consultant and the hospital nurse; and, by the same token, notes made by the nurse are kept with the doctor's records.

\section{New Schemes}

Even though there has been a great deal of experience in the launching of new attachment schemes it has always been felt to be very important that the initial discussions with the family doctors concerned should be by the county medical officer and the county nursing officer themselves, as the whole question of inaugurating a new attachment scheme can depend on the care with which it is prepared. A careful assessment is then made of the number of nursing staff required by a particular doctor or group of doctors by the rule-of-thumb method mentioned above. Before the attachment can be made final there is, from the administration point of view, detailed work which must be done; for the patients belonging to the particular practice will previously have been cared for by a variety of nurses, midwives, and health visitors, and they must now be adjusted to form the case-load of the particular nurse, midwife, and health visitor who will in future be working exclusively with the practice.

After the attachment scheme has been launched the county nursing officer and area nursing officers are in very much closer personal contact with the family doctors than previously. The area nursing officer continues to supervise the nursing staff and visit the family doctors at regular intervals to discuss with them how the scheme is working. This is particularly important in the early stages of a new attachment in order to ensure that the family doctors are well content with the new arrangements.

The area nursing officers must, of course, make full relief arrangements for off-duty and holidays for attached nursing staff, and this is catered for as part of the normal relief facilities available in the county. Sometimes, of course, in large group practices there are sufficient nurses, midwives, and health visitors attached to the firm for the nurses to be able to arrange for their own relief within the team.

\section{Premises}

So far as premises are concerned, in most cases family doctors arrange for child-welfare sessions to be held in their own surgeries one or two afternoons a week, with the attached health visitor in attendance together with the family doctor; similarly for antenatal sessions, relaxation sessions, and immunization sessions, again with the appropriate nurse or midwife in attendance. If the family doctor is unable to provide accommodation for such sessions then the county health department can arrange for premises to be made available to him without charge. But one is now beginning to find that attachments have been running for so long in this county that certain doctors when building new surgery accommodation have provided additions to allow for these services, and this is felt to be a very encouraging indication of the importance they place on this new way of working.

It is sometimes said that what can be done by way of nursing attachments in a county cannot be done in the town. This is not our experience in Hampshire; indeed, the most recent nursing-attachment scheme that has been launched in the county provides for health visitors and nurses for all family doctors working in a borough of some 80,000 population.

\section{Conclusion}

In conclusion, it is our experience in Hampshire that the greatest single contribution that can be made to assist the family doctor to give comprehensive medical care for all his patients is the attachment by local health authority of nurses, midwives, and health visitors to work with him and under his direction; it is certainly possible from the administrative point of view, and not nearly so costly to the local health authority as might be imagined. The Hampshire County Council accepts that for a far better medical service it is well content to meet the additional cost. 